\title{
Risk Factors That Increase Nosocomial Infections among Patients with Chest Tube
}

\author{
Maha Nafady Abd El Hafez, Hala Mohammed Ghanem \& Hussein El Khayat \\ Instructor of Medical-Surgical Nursing, Faculty of Nursing, Assiut University, Egypt \\ Assistant professor of Medical-Surgical Nursing, Faculty of Nursing, Assiut University, Egypt \\ Lecturer of Cardiothoracic-Surgery, Faculty of Medicine, Assiut University, Egypt
}

\begin{abstract}
Background: chest drains are used for patients who have undergone cardiothoracic surgery or chest trauma. It maintains cardiorespiratory function and hemodynamic stability by draining the pleural and mediastinal spaces of air, blood or other fluids. The aim of the study was to identify the risk factors that increase nosocomial infections among patients with a chest tube. A descriptive research design was used to conduct this study. The sample was consisted of a convenience sixty adult patients with a chest tube. The following tools were utilized for data collection: "Patient' Assessment Sheet", "Chest Tube' Assessment Sheet" and "Risk Factors of Nosocomial Infections' Assessment Sheet". Results concluded that; there was statistically significant difference between risk factors of nosocomial infection, the reason of insertion, type, place, site, number, the frequency of dressing and chest tube days. Conclusion: the most common risk factors were contaminated respiratory therapy equipment, mechanical ventilation, blood transfusion, mixed antibiotics, chronic illness, understaffing, overcrowding, improper hand washing and use of invasive devices. Recommendations: swabs should be taken from the site regularly and use aseptic technique when caring for patients to detect and prevent further infection.
\end{abstract}

\section{Keywords: Chest Tube, Risk Factors \& Nosocomial Infection.}

\section{Introduction}

Chest tube insertion is a procedure to place a flexible, hollow drainage tube into the chest in order to remove an abnormal collection of air or fluid from the pleural space (located between the inner and outer lining of the lung) (Laith et al.,2016).

Indications of chest tube include the following: - a collapsed lung, lung infection, bleeding around lung especially after trauma, fluid accumulation due to another medical condition such as cancer or pneumonia, an inability to breathe or difficulty breathing due to an accumulation of fluid or air. Surgery especially lung, heart or esophageal surgery. Inserting a chest tube may also help to diagnose other conditions, such as lung damage or internal injuries after trauma (Kane et al., 2013).

Contraindications to chest tube placement include refractory coagulopathy and presence of a diaphragmatic hernia, as well as hepatic hydrothorax. Additional contraindications include scarring in the pleural space (adhesions) and skin infection over the chest tube insertion site (Gil et al., 2014).

Chest tube insertion is one of the most dangerous, complicated and misunderstood procedures. Complications of tube thoracotomy can be classified as either technical or infective. Technical causes include tube malposition, blocked drain, chest drain dislodgement, re-expansion pulmonary edema, subcutaneous emphysema, nerve injuries, cardiac and vascular injuries, oesophageal injuries, residual/postextubation pneumothorax, fistulae, tumor recurrence at the insertion site, herniation through the site, chylothorax, and cardiac dysrhythmias. Infective complications include empyema and surgical site infection including cellulitis and necrotizing fasciitis (Kwiatt et al., 2014).

Infectious complications may develop in $2 \%$ to $25 \%$ of patients who undergo thoracotomy tube placement. Infection risk increases with duration of tube placement (Empyema-Chest tube (foreign object) could introduce bacteria into the pleural space). Infectious complications of chest tube insertion include pneumonia and positive bacterial cultures, empyema and surgical site infection including cellulitis and necrotizing fasciitis (Kesieme et al., 2011).

Mortality and morbidity from chest tube insertion are not strongly associated with the procedure itself. The primary cause responsible for fluid or air accumulation in the pleural cavity is related to continued illness and outcome such as pleural effusions caused by cancer. Chest tube insertion may be problematic in persons affected with certain connective tissue diseases (Laith et al., 2016).

A hospital-acquired infection also called a nosocomial infection (NI), is an infection that first appears between 48 hours and four days after a patient is admitted to a hospital or other health-care facility. A nosocomial infection is an infection whose development is favored by a hospital environment, such as one acquired by a patient during a hospital 
visit or one developing among hospital staff (Pollack, 2010).

Nosocomial infections occur worldwide, both in the developed and developing the world. They are a significant burden to patients and public health. They are a major cause of death and increased morbidity in hospitalized patients. They may cause increased functional disability and emotional stress and may lead to conditions that reduce the quality of life (Humphery-Smith, 2014).

The risk of infection among patients with chest tubes increases as the number of chest tube days increases. Patients who had chest tubes for more than 20 days were almost 6 times more likely to develop NI than those who had chest tubes for 20 days or less. Infection is likely to happen early during admission, which necessitates stringent adherence to infection control strategies, especially during that time frame (El-Masri et al., 2009).

\section{Significance of the study}

During the last year, according to hospital registry (cardiothoracic surgery department and trauma unit), about 700 patient underwent insertion of a chest tube in Assiut university hospital. During the training in Cardiothoracic surgery department, the researcher observed that patients with chest tube are at high risk of developing a nosocomial infection(NI), which then leads to higher mortality rates, prolonged hospitalization, as well increase the costs of treatment.

\section{Aim of the study}

The aim of this study is to identify the risk factors that increase nosocomial infections (NI) among patients with a chest tube.

\section{Subjects \& methods}

\section{Research design}

A descriptive research design utilized to conduct this study

\section{Research Question}

What are the risk factors leading to increasing nosocomial infections (NI) among patients with chest tube?

\section{I: Technical design \\ Setting}

This study was carried out in postoperative intensive care unit, cardiothoracic surgery department and trauma unit at Assiut university hospital.

\section{Subjects}

A sample of convenience sixty adult patients (included postoperative cardiothoracic surgery and trauma patients) with chest tube who were willing to participate in the study.

\section{Tools}

Data pertinent to the study were collected, utilizing the following three tools that developed by the researcher based on recent relevant review to achieve the purpose of the current study.

\section{Tool (1): Patient assessment sheet}

This tool was developed and modified by the researcher based on the recent literature review to assess patient with chest tube after admission (cardiothoracic surgery department, intensive care unit and trauma unit).It was constructed based on the following two parts:

Part one: it included socio-demographic data of the patients as (name, age, sex, marital status, occupation, the level of education, social status, date of admission and date of discharge). It included (9) items.

Part two: General assessment, this part included medical history (the past and present health history), Physical examination, Laboratory investigations and diagnostic procedures, Postoperative cardiothoracic surgery, and trauma complications. It included (4) items.

Tool (2): Chest tube assessment sheet

This tool was developed by the researcher to assess any abnormalities in patients with a chest tube. This sheet included the following three parts.

Part one: it included information of chest tube (date of insertion and removal, site, type, number, size and place of insertion).It included (7) items.

Part two: it included assessment of drainage system which constructed based on an assessment of nature of drainage fluid and presence of disconnection. It included (2) items.

Part three: It was concerned with the assessment of site of operation includes the following (frequency of dressing, signs and symptoms of localized and generalized infection of the skin). It included (2) items.

Tool (3): Assessment sheet of risk factors of nosocomial infections:

This tool was developed by the researcher to identify the risk factors that increase nosocomial infections among patients with chest tube that develops more than 48 hours after hospital admission. It included (4) items.

II: Administrative design

A letter was issued from the faculty of nursing at Assiut University to the head of cardiothoracic surgery department, the head of postoperative intensive care unit and trauma unit at Assiut University hospital explaining the purpose of the study and requesting their permission for collecting the data.

\section{III: Operational design}

This study was carried out in two phases: 


\section{Phase I: preparatory phase}

A review of current and past, local and international related literature in the various aspects using books, articles, periodicals, and magazines were done. The proposed study settings were assessed for the number of patients admitted to post-operative care unit, trauma unit and cardiothoracic surgery department who had a chest tube . This phase ended by contents validity and pilot study.

\section{Content validity}

The content validity was done by 5 expertise (medical staff) from cardiothoracic surgery department \& (nursing staff) from the medicalsurgical nursing who reviewed the tools for clarity, relevance, comprehensiveness, understanding, applicability and easiness for administrative minor modifications were required and correction was carried out accordingly. Then the final form of the tools was designed and tested for reliability by using internal consistency for the tools measured using Cronbach test, the tools proved to be reliable (0.73).

\section{A pilot study}

A Pilot study was conducted on $10 \%$ of the sample (6 patients) admitted to the postoperative intensive care unit (ICU), trauma unit and cardiothoracic surgery department to test the feasibility and clarity of the tools. According to this pilot study, the required modifications were made. Those patients who were involved in the pilot study were included in the study. The purpose of the pilot study was: To ascertain the relevance of the tool, detect any problem might interfere with the process of data collection and to estimate the time needed to complete the study.

\section{Phase II: implementation phase}

Data were collected at the cardiothoracic surgery department, postoperative intensive care unit and trauma unit at Assiut University Hospital during the period from 1-9-2015 to 1-2-2016; the tools were all filed through interviewing. The purpose of the study was explained to all patients prior to collection of data. The study was carried out during morning shift for all available patients.

Once the permission was granted to proceed with the proposed study ,the research proposal was submitted to the research committee in Assiut university hospital ,name of potential patients who have admitted to the units and who met the criteria were obtained daily from the nurses in cardiothoracic surgery department, post-operative intensive care unit and trauma unit in the hospital.

After taking the patient oral agreement for voluntary participation in the study, each patient was contacted personally by the researcher, then the purpose and the nature of the study were explained, then fills the patient's structured assessment sheet, chest tube sheet and risk factors of nosocomial infection sheet through the following:

- The baseline data were collected such as pain assessment and its site.

- Assessment of the respiratory system as respiratory rate, depth, cough, sputum, pain with breathing.

- Assess skin color and status of surgical dressing.

- Monitor characteristics of drainage including color, amount and consistency.

- Monitor the patient for the signs and symptoms of infection.

- Assess vital signs for any abnormality until chest tube removal.

- Review patient's record for the result of the laboratory investigations and diagnostic studies.

Ethical and legal consideration

Confidentiality and anonymity of patients were assured through coding of all data. Each patient has right to withdraw each from the study at any time without any rational.

\section{Limitation of the study}

1. Diagnostic studies and laboratory investigations which were important to make comparisons between patients on the day of chest tube insertion and changes happen to them until tube removal were done for most patients on admission only.

2. Vital signs were not recorded in patient's charts in cardiothoracic surgery department and trauma unit which are very important to detect signs of infection.

3. Anthropometric measurements weren't done for all patients to detect patients with risk for malnutrition or infection. 


\section{Results}

Table (1):- Percentage distribution of studied patients as regarding their socio-demographic characteristics $(\mathrm{n}=60)$.

\begin{tabular}{|c|c|c|}
\hline \multirow{2}{*}{ Characteristics } & \multicolumn{2}{|c|}{ Frequency } \\
\hline & No. & $\%$ \\
\hline $\begin{array}{ll}\text { Age } & \\
\text { 1. } 18<30 \text { years } \\
\text { 2. } 30<45 \text { years } \\
\text { 3. } 45-65 \text { years } \\
\end{array}$ & $\begin{array}{l}26 \\
15 \\
19\end{array}$ & $\begin{array}{l}43.3 \\
25.0 \\
31.7 \\
\end{array}$ \\
\hline Mean+SD & \multicolumn{2}{|c|}{$37.1+11.4$} \\
\hline $\begin{array}{lll}\text { Sex } & \\
& \bullet \text { Male } \\
& \bullet \text { Female }\end{array}$ & $\begin{array}{c}52 \\
8\end{array}$ & $\begin{array}{l}86.7 \\
13.3\end{array}$ \\
\hline 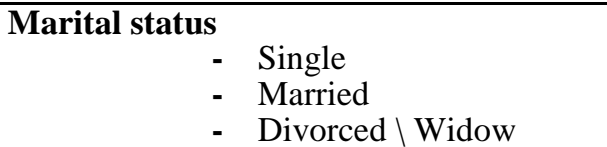 & $\begin{array}{c}26 \\
33 \\
1\end{array}$ & $\begin{array}{c}43.3 \\
55.0 \\
1.7\end{array}$ \\
\hline $\begin{array}{l}\text { Level of education } \\
\qquad \begin{aligned} \bullet & \text { Illiterate } \\
\bullet & \text { Primary } \\
\bullet & \text { Intermediate } \\
\bullet & \text { High }\end{aligned}\end{array}$ & $\begin{array}{c}16 \\
5 \\
33 \\
6\end{array}$ & $\begin{array}{c}26.7 \\
8.3 \\
55.0 \\
10.0\end{array}$ \\
\hline $\begin{array}{ccc}\text { Occupation } & \\
& \bullet & \text { No-working } \\
& \bullet & \text { Constant salary } \\
& \bullet & \text { Inconstant salary } \\
\end{array}$ & $\begin{array}{c}32 \\
7 \\
21\end{array}$ & $\begin{array}{l}53.3 \\
11.7 \\
35.0\end{array}$ \\
\hline $\begin{array}{l}\text { Social status -Family size } \\
\qquad \begin{aligned} \bullet & <\text { person } \\
\bullet & 4-8 \text { person } \\
\bullet & 9 \text { and more person }\end{aligned}\end{array}$ & $\begin{array}{c}0 \\
49 \\
11\end{array}$ & $\begin{array}{c}0.0 \\
81.7 \\
18.3\end{array}$ \\
\hline $\begin{aligned} \text { Hospital stay per week } \\
\bullet \quad<\text { week } \\
\bullet \quad \text { One week } \\
\bullet \quad \text { More than week }\end{aligned}$ & $\begin{array}{c}33 \\
6 \\
21\end{array}$ & $\begin{array}{l}55.0 \\
10.0 \\
35.0\end{array}$ \\
\hline
\end{tabular}

Table (2): Percentage distribution of the studied patients as regarding present health history and presence of complications $(\mathbf{n}=\mathbf{6 0})$.

\begin{tabular}{|c|c|c|}
\hline \multirow{2}{*}{ Items } & \multicolumn{2}{|c|}{ Frequency } \\
\hline & No. & $\%$ \\
\hline \multicolumn{3}{|l|}{ Present health history (reason of insertion): } \\
\hline 1. Chest trauma & 31 & \\
\hline 2. Postoperative cardiac surgery & 10 & 16.7 \\
\hline 3. Infected pleural cavity & 9 & 15.0 \\
\hline$\bullet \quad$ Empyema & 5 & 8.3 \\
\hline - $\quad$ Lung abscess & 4 & 6.7 \\
\hline 4. Non-infected pleural cavity & 10 & 16.7 \\
\hline - Pneumothorax & 5 & 8.3 \\
\hline - Hemothorax & 1 & 1.7 \\
\hline - Hemopneumothorax & 0 & 0.0 \\
\hline - Others (pleural effusion) & 4 & 6.7 \\
\hline \multirow{2}{*}{$\frac{\text { Postoperative cardiothoracic surgery complications }}{\bullet \text { Infection }}$} & & \\
\hline & 7 & 11.7 \\
\hline \multicolumn{3}{|l|}{ Trauma complications } \\
\hline$\bullet \quad$ wound infection & 1 & 1.7 \\
\hline
\end{tabular}


Table (3): Percentage distribution of the studied patients as criteria of chest tube and drainage system ( $n=60)$

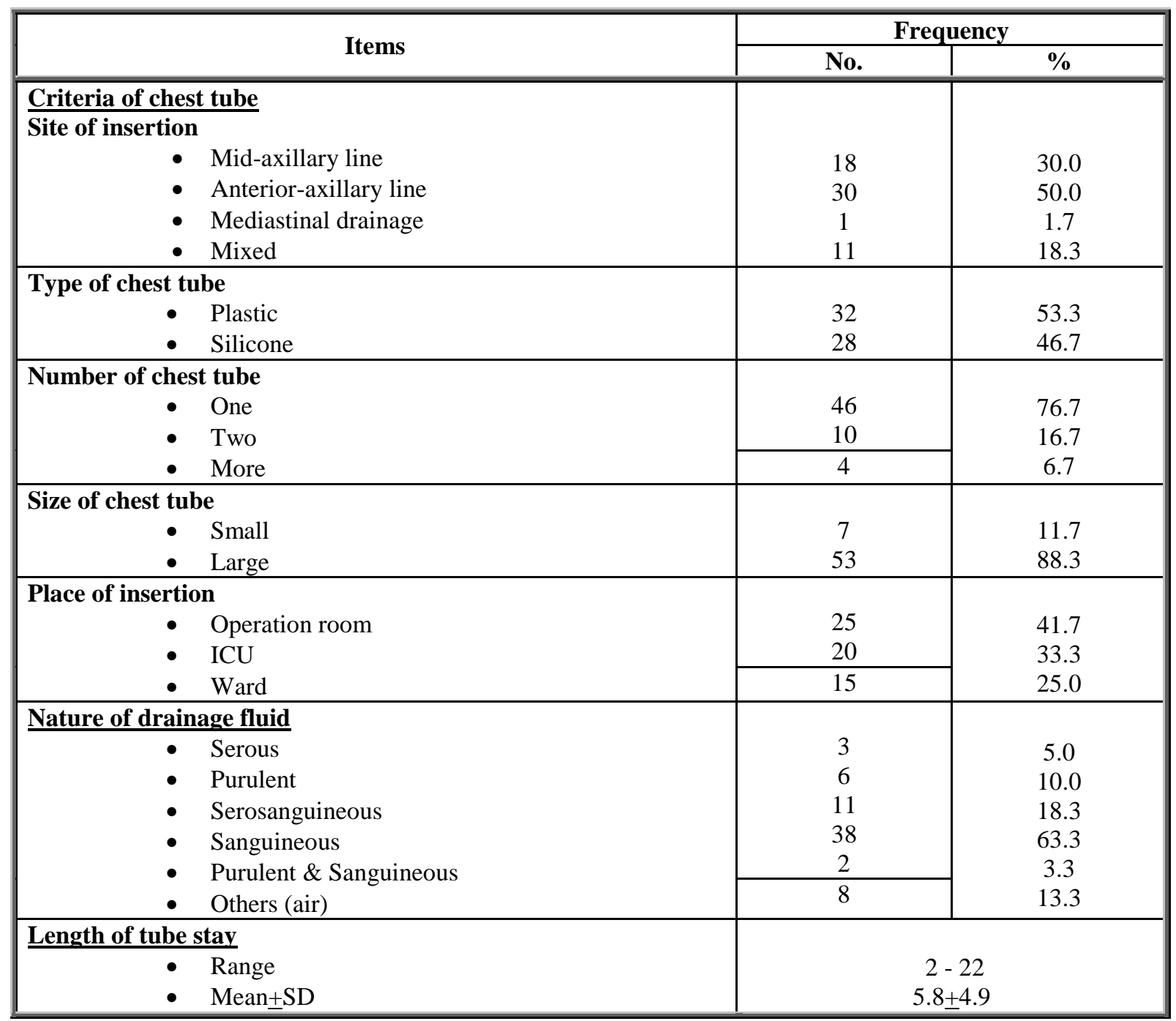

Table (4): Percentage distribution of risk factors for nosocomial infection among patients with chest tube insertion $(n=60)$.

\begin{tabular}{|c|c|c|}
\hline \multirow{2}{*}{ Risk factors for nosocomial infection } & \multicolumn{2}{|c|}{ Frequency } \\
\hline & No. & $\%$ \\
\hline \multicolumn{3}{|l|}{ 1-Respiratory tract nosocomial infection } \\
\hline$\bullet$ Contaminated respiratory therapy equipment & 15 & 25.0 \\
\hline - Patient on mechanical ventilation & 9 & 15.0 \\
\hline \multicolumn{3}{|l|}{ Hours of mechanical ventilation } \\
\hline-4 hours & 4 & 6.7 \\
\hline - 8 hours & 2 & 3.3 \\
\hline - 24 hours & 3 & 5.0 \\
\hline \multicolumn{3}{|l|}{ 2-Blood stream nosocomial infectio } \\
\hline - $\quad$ Blood transfusions were given & 27 & 45.0 \\
\hline - Improper care of needle insertion site & 7 & 11.7 \\
\hline - Failure to change I.V access site & 1 & 1.7 \\
\hline 3-Surgical or traumatic wound nosocomial infection & & \\
\hline$\bullet \quad$ Failure to use aseptic technique during dressing & 1 & 1.7 \\
\hline
\end{tabular}




\begin{tabular}{|l|c|c|}
\hline \multicolumn{1}{|c|}{ Risk factors for nosocomial infection } & \multicolumn{2}{|c|}{ Frequency } \\
\cline { 2 - 3 } & \multicolumn{2}{|c|}{ No. } \\
\hline 4-Other general risk factors & & \\
\hline - Presence of pre-existing infection & 5 & 8.3 \\
- Use of antibiotics & 41 & 68.3 \\
- Use of anticoagulant & 13 & 21.7 \\
- Chronic illness & 31 & 51.7 \\
- Poor nutritional status & 19 & 31.7 \\
- Understaffing & 44 & 73.3 \\
- Overcrowding & 44 & 73.3 \\
- Smoking & 27 & 45.0 \\
- Prolonged hospital stay & 23 & 38.3 \\
- Improper hand washing & 49 & 81.7 \\
\hline
\end{tabular}

Table (5): Percentage distribution of the studied patients in the relationship between risk factors of nosocomial infection and age $(n=60)$.

\begin{tabular}{|c|c|c|c|c|c|c|c|}
\hline \multirow[t]{2}{*}{ Risk factors } & \multicolumn{2}{|c|}{ 18-30 years } & \multicolumn{2}{|c|}{$\begin{array}{c}\text { Age } \\
\text { 30-44 years }\end{array}$} & \multicolumn{2}{|c|}{ 44-65 years } & \multirow[t]{2}{*}{ P. value } \\
\hline & No & $\%$ & No & $\%$ & No & $\%$ & \\
\hline \multicolumn{8}{|l|}{ 1- Respiratory tract infection } \\
\hline Contaminated respiratory therapy equipment & 5 & 8.3 & 3 & 5.0 & 7 & 11.7 & \multirow{2}{*}{0.195} \\
\hline Patient on mechanical ventilation & 2 & 3.3 & 5 & 8.3 & 2 & 3.3 & \\
\hline \multicolumn{8}{|l|}{ Hours of mechanical ventilation } \\
\hline 4 hours & 1 & 1.7 & 2 & 3.3 & 1 & 1.7 & \multirow{3}{*}{0.717} \\
\hline 8 hours & 0 & 0.0 & 1 & 1.7 & 1 & 1.7 & \\
\hline 24 hours & 1 & 1.7 & 2 & 3.3 & 0 & 0.0 & \\
\hline \multicolumn{8}{|l|}{ 2-Blood stream infection } \\
\hline Blood transfusions given & 12 & 20.0 & 6 & 10.0 & 9 & 15.0 & \multirow{3}{*}{0.435} \\
\hline Improper care of needle insertion site & 2 & 3.3 & 3 & 5.0 & 2 & 3.3 & \\
\hline Failure to change I.V access site & 0 & 0.0 & 1 & 1.7 & 0 & 0.0 & \\
\hline \multicolumn{8}{|c|}{ 3-Surgical or traumatic wound nosocomial infection } \\
\hline Failure to use aseptic technique during & 1 & 1.7 & 0 & 0.0 & 0 & 0.0 & - \\
\hline \multicolumn{8}{|l|}{ 4-Others } \\
\hline Presence of pre-existing infection & 1 & 1.7 & 1 & 1.7 & 3 & 5.0 & \multirow{11}{*}{0.647} \\
\hline Use of antibiotics & 16 & 26.7 & 10 & 16.7 & 15 & 25.0 & \\
\hline Use of anticoagulant & 5 & 8.3 & 5 & 8.3 & 3 & 5.0 & \\
\hline Chronic illness & 10 & 16.7 & 9 & 15.0 & 12 & 20.0 & \\
\hline Poor nutritional status & 9 & 15.0 & 6 & 10.0 & 4 & 6.7 & \\
\hline Understaffing & 23 & 38.3 & 9 & 15.0 & 12 & 20.0 & \\
\hline Overcrowding & 23 & 38.3 & 9 & 15.0 & 12 & 20.0 & \\
\hline Smoking & 10 & 16.7 & 7 & 11.7 & 10 & 16.7 & \\
\hline Prolonged hospital stay & 8 & 13.3 & 6 & 10.0 & 9 & 15.0 & \\
\hline Improper hand washing & 24 & 40.0 & 9 & 15.0 & 16 & 26.7 & \\
\hline Use of other invasive devices & 9 & 15.0 & 7 & 11.7 & 9 & 15.0 & \\
\hline
\end{tabular}


Table (6): Percentage distribution of the studied patients in relationship between risk factors of nosocomial infection and reason of insertion of a chest tube.

\begin{tabular}{|c|c|c|c|c|c|c|c|c|c|}
\hline \multirow[t]{2}{*}{ Risk factors } & \multicolumn{2}{|c|}{ Chest trauma } & \multicolumn{2}{|c|}{$\begin{array}{c}\text { Post } \\
\text { operative } \\
\text { cardiac } \\
\text { surgery }\end{array}$} & \multicolumn{2}{|c|}{$\begin{array}{c}\text { Infected } \\
\text { pleural } \\
\text { cavity }\end{array}$} & \multicolumn{2}{|c|}{$\begin{array}{l}\text { Non-infected } \\
\text { pleural cavity }\end{array}$} & \multirow[t]{2}{*}{$\begin{array}{c}\text { P. } \\
\text { Value }\end{array}$} \\
\hline & No & $\%$ & No & $\%$ & No & $\%$ & No & $\%$ & \\
\hline \multicolumn{10}{|l|}{ 1- Respiratory infection } \\
\hline $\begin{array}{l}\text { Contaminated respiratory therapy } \\
\text { equipment }\end{array}$ & 6 & 10.0 & 0 & 0 & 4 & 6.7 & 11 & 18.3 & \multirow{2}{*}{$\begin{array}{c}<0.00 \\
1 * *\end{array}$} \\
\hline Patient on mechanical ventilation & 0 & 0 & 9 & 15 & 0 & 0 & 0 & 0 & \\
\hline \multicolumn{10}{|l|}{ 2-Blood stream infection } \\
\hline Blood transfusions given & 10 & 16.7 & 9 & 15 & 5 & 8.3 & 14 & 31.6 & \multirow{2}{*}{0.515} \\
\hline Improper care of needle site & 5 & 8.3 & 1 & 1.7 & 2 & 3.3 & 3.0 & 5.0 & \\
\hline \multicolumn{10}{|l|}{ 3-Surgical or wound infection } \\
\hline Failure to use aseptic technique. & 1 & 1.7 & 0 & 0.0 & 0 & 0.0 & 0 & 0.0 & - \\
\hline \multicolumn{10}{|l|}{ 4- Other general risk factors } \\
\hline Presence of pre-existing infection & 0 & 0 & 2 & 3.3 & 3 & 5.0 & 0 & 0 & \multirow{11}{*}{$\begin{array}{c}<0.00 \\
1 * *\end{array}$} \\
\hline Use of antibiotics & 14 & 23.3 & 10 & 16.7 & 9 & 15 & 23 & 38.3 & \\
\hline Use of anticoagulant & 4 & 6.7 & 8 & 13.3 & 0 & 0 & 5 & 8.3 & \\
\hline Chronic illness & 10 & 16.7 & 9 & 15.0 & 7 & 11.7 & 15 & 25.0 & \\
\hline Poor nutritional status & 7 & 11.7 & 5 & 8.3 & 5 & 8.3 & 9 & 15.0 & \\
\hline Understaffing & 31 & 51.7 & 0 & 0 & 6 & 10 & 37 & 61.7 & \\
\hline O/vercrowding & 31 & 51.7 & 0 & 0 & 5 & 8.3 & 38 & 63.3 & \\
\hline +Smoking & 12 & 20 & 3 & 5.0 & 7 & 11.7 & 17 & 28.3 & \\
\hline Prolonged hospital stay & 6 & 10.0 & 3 & 5.0 & 8 & 11.7 & 13 & 21.7 & \\
\hline Improper hand washing & 29 & 48.3 & 4 & 6.7 & 9 & 15.0 & 36 & 60.0 & \\
\hline Use of other invasive devices & 11 & 18.3 & 10 & 16.7 & 3 & 5.0 & 12 & 20.0 & \\
\hline
\end{tabular}

Table (7): Percentage distribution of the studied patients in the relationship between risk factors for nosocomial infection and a number of a chest tube.

\begin{tabular}{|c|c|c|c|c|c|c|c|}
\hline \multirow{3}{*}{ Risk factors } & \multicolumn{6}{|c|}{ Number of chest tube } & \multirow{3}{*}{ P. value } \\
\hline & \multicolumn{2}{|c|}{ One } & \multicolumn{2}{|c|}{ Two } & \multicolumn{2}{|c|}{ More } & \\
\hline & No & $\%$ & No & $\%$ & No & $\%$ & \\
\hline \multicolumn{8}{|l|}{ 1- Respiratory tract nosocomial infection } \\
\hline Contaminated respiratory therapy equipment & 12 & 20.0 & 2 & 3.3 & 1 & 1.7 & \multirow{2}{*}{$0.001 * *$} \\
\hline Patient on mechanical ventilation & 0 & 0.0 & 6 & 10.0 & 3 & 5.0 & \\
\hline \multicolumn{8}{|l|}{ 2-Blood stream nosocomial infection } \\
\hline Blood transfusions given & 15 & 25.0 & 8 & 13.3 & 4 & 6.7 & \multirow{3}{*}{0.468} \\
\hline Improper care of needle insertion site & 5 & 8.3 & 2 & 3.3 & 0 & 0.0 & \\
\hline Failure to change I.V access site & 0 & 0.0 & 1 & 1.7 & 0 & 0.0 & \\
\hline \multicolumn{8}{|c|}{ 3-Surgical or traumatic wound nosocomial infection } \\
\hline Failure to use aseptic technique during & 1 & 1.7 & 0 & 0.0 & 0 & 0.0 & - \\
\hline \multicolumn{8}{|l|}{ 4-Others } \\
\hline Presence of pre-existing infection & 2 & 3.3 & 3 & 5.0 & 0 & 0.0 & \multirow{11}{*}{$0.008 * *$} \\
\hline Use of antibiotics & 28 & 46.7 & 9 & 15.0 & 4 & 6.7 & \\
\hline Use of anticoagulant & 5 & 8.3 & 5 & 8.3 & 3 & 5.0 & \\
\hline Chronic illness & 18 & 30.0 & 9 & 15.0 & 4 & 6.7 & \\
\hline Poor nutritional status & 11 & 18.3 & 5 & 8.3 & 3 & 5.0 & \\
\hline Understaffing & 40 & 66.7 & 4 & 6.7 & 0 & 0.0 & \\
\hline Overcrowding & 40 & 66.7 & 4 & 6.7 & 0 & 0.0 & \\
\hline Smoking & 22 & 36.7 & 4 & 6.7 & 1 & 1.7 & \\
\hline Prolonged hospital stay & 17 & 28.3 & 4 & 6.7 & 2 & 3.3 & \\
\hline Improper hand washing & 41 & 68.3 & 6 & 10 & 2 & 3.3 & \\
\hline Use of other invasive devices & 16 & 26.7 & 6 & 10 & 3 & 5.0 & \\
\hline
\end{tabular}


Table (8): Percentage distribution of the studied patients in the relationship between risk factors for nosocomial infection and size of a chest tube.

\begin{tabular}{|c|c|c|c|c|c|}
\hline \multirow{3}{*}{ Risk factors } & \multicolumn{4}{|c|}{ Size of chest tube } & \multirow{3}{*}{ P. value } \\
\hline & \multicolumn{2}{|c|}{ Small } & \multicolumn{2}{|c|}{ Large } & \\
\hline & No & $\%$ & No & $\%$ & \\
\hline \multicolumn{6}{|l|}{ 1- Respiratory tract nosocomial infection } \\
\hline Contaminated respiratory therapy equipment & 2 & 3.3 & 13 & 21.7 & \multirow{2}{*}{0.252} \\
\hline Patient on mechanical ventilation & 0 & 0.0 & 9 & 15.0 & \\
\hline \multicolumn{6}{|l|}{ 2-Blood stream nosocomial infection } \\
\hline Blood transfusions given & 1 & 1.7 & 26 & 43.3 & \multirow{3}{*}{0.858} \\
\hline Improper care of needle insertion site & 0 & 0.0 & 7 & 11.7 & \\
\hline Failure to change I.V access site & 0 & 0.0 & 1 & 1.7 & \\
\hline \multicolumn{6}{|c|}{ 3-Surgical or traumatic wound nosocomial infection } \\
\hline Failure to use aseptic technique during & 0 & 0.0 & 1 & 1.7 & - \\
\hline \multicolumn{6}{|l|}{ 4-Others } \\
\hline Presence of pre-existing infection & 0 & 0.0 & 5 & 8.3 & \multirow{11}{*}{0.523} \\
\hline Use of antibiotics & 3 & 5.0 & 38 & 63.3 & \\
\hline Use of anticoagulant & 1 & 1.7 & 12 & 20.0 & \\
\hline Chronic illness & 2 & 3.3 & 29 & 48.3 & \\
\hline Poor nutritional status & 0 & 0.0 & 19 & 31.7 & \\
\hline Understaffing & 6 & 10.0 & 38 & 63.3 & \\
\hline Overcrowding & 6 & 10.0 & 38 & 63.3 & \\
\hline Smoking & 3 & 5.0 & 24 & 40.0 & \\
\hline Prolonged hospital stay & 2 & 3.3 & 21 & 35.0 & \\
\hline Improper hand washing & 5 & 8.3 & 44 & 73.3 & \\
\hline Use of other invasive devices & 0 & 0.0 & 25 & 41.7 & \\
\hline
\end{tabular}

Table (9): Percentage distribution of the studied patients in the relationship between risk factors for nosocomial infection and duration of chest tube stay.

\begin{tabular}{|c|c|c|c|c|c|c|c|}
\hline \multirow{2}{*}{ Risk factors } & \multicolumn{6}{|c|}{ Duration of stay } & \multirow{2}{*}{ P. value } \\
\hline & \multicolumn{2}{|c|}{ 2-3 days } & \multicolumn{2}{|c|}{ 4-6 days } & \multicolumn{2}{|c|}{7 days or more } & \\
\hline \multicolumn{8}{|l|}{ 1- Respiratory tract nosocomial infection } \\
\hline Contaminated respiratory therapy equipment & 4 & 6.7 & 1 & 1.7 & 10 & 16.7 & \multirow{2}{*}{$0.010 * *$} \\
\hline Patient on mechanical ventilation & 3 & 5.0 & 5 & 8.3 & 1 & 1.7 & \\
\hline \multicolumn{7}{|l|}{ Hours of mechanical ventilation } & \multirow{4}{*}{0.367} \\
\hline 4 hours & 0 & 0.0 & 3 & 5.0 & 1 & 1.7 & \\
\hline 8 hours & 1 & 1.7 & 1 & 1.7 & 0 & 0.0 & \\
\hline 24 hours & 2 & 3.3 & 1 & 1.7 & 0 & 0.0 & \\
\hline \multicolumn{8}{|l|}{ 2-Blood stream nosocomial infection } \\
\hline Blood transfusions given & 11 & 18.3 & 7 & 11.7 & 9 & 15.0 & \multirow{3}{*}{0.646} \\
\hline Improper care of needle insertion site & 4 & 6.7 & 3 & 5.0 & 7 & 11.7 & \\
\hline Failure to change I.V access site & 1 & 1.7 & 1 & 1.7 & 0 & 0.0 & \\
\hline \multicolumn{8}{|l|}{ 3-Surgical or traumatic wound infection } \\
\hline Failure to use aseptic technique during & 1 & 1.7 & 1 & 1.7 & 0 & 0.0 & - \\
\hline \multicolumn{8}{|l|}{ 4-Others } \\
\hline Presence of pre-existing infection & 2 & 3.3 & 2 & 3.3 & 1 & 1.7 & \multirow{5}{*}{0.546} \\
\hline Use of antibiotics & 12 & 20.0 & 13 & 21.7 & 16 & 26.7 & \\
\hline Use of anticoagulant & 4 & 6.7 & 5 & 8.3 & 4 & 6.7 & \\
\hline Chronic illness & 10 & 16.7 & 10 & 16.7 & 11 & 18.3 & \\
\hline Poor nutritional status & 8 & 13.3 & 5 & 8.3 & 6 & 10.0 & \\
\hline
\end{tabular}




\begin{tabular}{|c|c|c|c|c|c|c|c|}
\hline \multirow{3}{*}{ Risk factors } & \multicolumn{6}{|c|}{ Duration of stay } & \multirow{8}{*}{ P. value } \\
\hline & \multicolumn{2}{|c|}{ 2-3 days } & \multicolumn{2}{|c|}{ 4-6 days } & \multicolumn{2}{|c|}{7 days or more } & \\
\hline & 23 & 38.3 & 6 & 10.0 & 15 & 25.0 & \\
\hline Overcrowding & 22 & 36.7 & 7 & 11.7 & 15 & 25.0 & \\
\hline Smoking & 13 & 21.7 & 6 & 10.0 & 8 & 13.3 & \\
\hline Prolonged hospital stay & 5 & 8.3 & 4 & 6.7 & 14 & 23.3 & \\
\hline Improper hand washing & 23 & 38.3 & 11 & 18.3 & 15 & 25.0 & \\
\hline Use of other invasive devices & 13 & 21.7 & 7 & 11.7 & 5 & 8.3 & \\
\hline
\end{tabular}

Table (1): shows that approximately half of the studied patients $(43.3 \%)$ their age ranged from $(18<30$ years) and the mean age was $(37.1 \pm 11.4)$. the majority of them $(86.7 \%)$ were male. More than half $(55 \%)$ of patients were married with intermediate education. As regard occupation, more than half $(53.3 \%)$ had no working. The majority $(81.7 \%)$ their family size was ranged between (4-8 persons). As regards hospital stay, more than half $(55 \%)$ of patients stayed less than one week and the mean of hospital stay was $(9 \pm 10.6)$.

Table (2): shows studied patients as regard present health history. It was noticed that the main cause of insertion of chest tube in more than half $(51.7 \%)$ of patients was chest trauma and the minority (1.7\%) of patients inserted a chest tube for evacuation of hemothorax. Regarding complications, the only complication that occurred was an infection in both postoperative cardiothoracic surgery patient $(11.7 \%)$ and trauma patient $(1.7 \%)$.

Table (3): shows that half $(50 \%)$ of patients inserted chest tube at anterior-axillary line. More than half $(53.3 \%)$ used a plastic chest tube. The majority $(76.7 \%)$ used one, large $(88.3 \%)$ chest tube and inserted it at the operation room $(41.7 \%)$. As regards to nature of drainage fluid, more than half $(63.3 \%)$ of patients their exudates were sanguineous. The mean of chest tube stay was $(5.8 \pm 4.9)$.

Table (4): shows that the main risk factors for respiratory tract nosocomial infection were contaminated respiratory therapy equipment (25\%) and patient on mechanical ventilation (15\%). Regarding blood stream nosocomial infection, the main risk factors were blood transfusions (45\%) and improper care of needle insertion site (11.7\%). Failure to use the aseptic technique during dressing $(1.7 \%)$ was the risk factors for surgical or traumatic wound nosocomial infection. As regards other general risk factors, the main risks were the use of mixed antibiotic, chronic illness, understaffing, overcrowding, smoking, improper hand washing and use of other invasive devices such as (central venous catheter, urinary catheter, and nasogastric tube).

Table (5): clarifies that, there was no statistical significance difference between risk factors of nosocomial infection and patients' age.
Table (6): shows that there was highly statistical significance difference between risk factors of respiratory tract nosocomial infection and reason of insertion of a chest tube and between other general risk factors and reason of insertion of a chest tube.

Table (7): illustrates that there was highly statistical significance difference between risk factors of respiratory tract nosocomial infection and a number of a chest tube and between other general risk factors and the number of a chest tube.

Table (8): shows that there was no statistical significance difference between risk factors and size of a chest tube.

Table (9): illustrates that there was highly statistical significance difference between risk factors of respiratory tract nosocomial infection and chest tube days, so infection increases with prolonged use of chest tube.

\section{Discussion}

Tube thoracotomy is widely used throughout the medical, surgical, and critical care specialties. It is generally used to drain pleural collections either as elective or emergency. Infectious complications may develop in $2 \%$ to $25 \%$ of patients who undergo thoracotomy tube placement. Infection risk increases with duration of tube placement (Empyema-Chest tube (foreign object) could introduce bacteria into the pleural space). Infectious complications of chest tube insertion include pneumonia and positive bacterial cultures, empyema and surgical site infection including cellulitis and necrotizing fasciitis (Kesieme et al., 2011).

Based on the results of the present study; the mean age was $(37.1 \pm 11.4)$; the majority of the patients were males. As regards the marital status, more than half were married with secondary education. These findings are in the same line with Olgac et al., (2006) who reported that the majority of patients were male with a mean age of $34.2 \pm 9.7$.

The present study disagrees with Abd El Hafez (2010) who was found that the mean age was (27.75 \pm $12.12)$ in the study group and $(26.20 \pm 17.65)$ in the control group. The majority of patients were females, single and illiterate. 
Oldfield et al., (2009) disagree with the current study as regard the mean age of patients which was 60.26 years, but he agrees with the study as regard to sex which revealed that the majority of patients were male .

Oldfield et al., (2009) reported that the mean outcome length of hospital stay was 7.7 days (range 1 to 59 days). This was confirmed by the result of the present study which illustrated that the mean of hospital stay was $(9+10.6)$ (range from 2 to 72 days).

As regarding to reason of insertion of the chest tube, the present study revealed that the main cause of insertion of chest tube in majority of patients was chest trauma, then as a consequence of postoperative cardiothoracic surgery and non-infected pleural cavity (include pneumothorax, hemothorax, hemopneumothorax and pleural effusion) and finally as a result of infected pleural cavity which varies between empyema and lung abscess. These results disagree with Oldfield et al., (2009) who reported that the majority of patients had a chest tube inserted during the performance of a surgical procedure,then trauma patients and the remaining chest tubes were inserted for treatment of pneumothorax, pleural effusion, pneumohemothorax, and hemothorax.

The present study was found that majority of study group inserted chest tube at anterior-axillary line. The majority used a silicone, large one chest tube and inserted it at operation room. This was confirmed by Oldfield (2005) who reported that a large sized chest tube was inserted as $72.5 \%$ of the patients had tubes sized 26 or greater.

Abd El Hafez (2010) noticed that more than half of patients on study and control groups had 3 chest tube. In relation to site of insertion of chest tube, it was founded that the majority of patients in study and control groups had mid-axillary chest tube and anterior-axillary chest tube.

As regarding nature of drainage fluid, the present study founded that more than half of patients their exudates were sanguineous, when the drainage observed for the first time. Carlson \& JynnMchale., (2009) reported that an important role of the nurse is to assess the chest tubes regularly every 2 hours for patency, fluctuation, a mount, consistency \&character of drainage

The present study revealed that the mean of chest tube stay was $(5.8 \pm 4.9)$.This result disagree with Oldfield et al., (2009) who was found that patients had a mean number of chest tube days of 18.8 (range 1 to 120 days). Eisenberg \& Khabbaz, (2011) added that the water seal group lasted a mean of 1.5 days \pm 0.3 days.

The present study illustrated that the main risk factors for nosocomial infection were blood transfusion, use of mixed antibiotic, chronic illness, poor nutritional status, understaffing, overcrowding, smoking, improper hand washing, prolonged hospital stay, mechanical ventilation and use of other invasive devices such as (central venous catheter, urinary catheter and nasogastric tube).This study was in the same line with Oldfield et al., (2009) who was founded that the majority of patients required a central venous catheter $(74.2 \%)$, while $13.3 \%$ required transfusion of at least one unit of blood and that the use of mechanical ventilation increased the risk of developing hospital-acquired infection by almost five times.

Lindgren \& Ames., (2005) Added that ventilated patients after thoracotomy have a significant risk of developing a nosocomial infection as a result of suppressed immune function and the presence of artificial tube.

Athanassiadi (2007) mentioned that heavy smoking, previous stroke, severe chronic lung disease, prior radiation therapy and underlying chronic medical problems (such as heart disease, renal failure, uncontrolled diabetes, HIV, immunodeficiency) are considered risk factors for nosocomial infections.

Gastmeier et al., (2007) mentioned that many patient characteristics and significant risk factors for ventilator-associated pneumonia had been included age, male gender, hospitalization prior to ICU admission, length of ICU stay, treatment in large hospitals, a low Glasgow Coma Scale (GCS), respiratory failure, congestive heart failure, acute renal failure and dialysis, bronchoscopy, tracheotomy, re-intubation, duration of mechanical ventilation, detection of certain multi-drug resistant pathogens, use of central vein catheters, bacteremia, enteral feeding, and application of corticosteroids.

Kwiatt et al., (2014) mentioned that closed tube thoracotomy is classified as "clean contaminated" and hence risk of infection of a wound is $7.7 \%$. By acting as a foreign body, the tube may provide a "common plane" for infectious exposure between the insertion site, the chest wall, and the pleural space. Necrotizing fasciitis, a rapidly progressive, lifethreatening infection of subcutaneous tissue and fascia has been reported in association with tube placement.

The present study revealed that $(16.7 \%)$ of study group developed a nosocomial infection (wound or surgical site infection) which disagree with Oldfield et al., (2009) who found that 40 cases developed nosocomial infections, minimaly had blood stream infection, while the majority of patients had pneumonia.

The present study found that there was no statistical significance difference between risk factors and patients' age. This study in the same line with Pekka, (2006) as conclusion of his study was that age and 
gender were not risked factors for a nosocomial infection and disagree with Gastmeier et al., (2007) who found that many patient characteristics and significant risk factors for ventilator-associated pneumonia included age and male gender.

Blot et al., (2014) added that incidence of nosocomial infections gradually increases in patients over 65 year age population. There is a significant relationship between increased age and predisposition to nosocomial infections. Predisposition to infections in this age group is a result of impaired host defense, underlying chronic diseases, long-term hospitalization, steroids and immunosuppressive therapies and malnutrition.

The present study revealed that there was highly statistical significance difference between risk factors and reason of insertion of a chest tube. This result in the same line with Tukenmez Tigen et al., (2014) who reported that trauma patients tended to develop NI more than non-trauma patients.

El-Masri et al., (2009) reported that trauma patients were reported to be at increased risk for NI because of unclean penetrating injuries, trauma-related depression of humeral and cell-mediated responses, massive blood loss and blood transfusion, inadequate nutritional support and the extensive use of invasive devices. A study examining the differences in NI in trauma and surgical patients reported that the overall infection rate among trauma patients was $11.64 \%$ compared with $6.43 \%$ for surgical patients. Trauma patients were reported to have higher rates of VAP and NBSI.

The present study found that there was highly statistical significance difference between risk factors, type and number of a chest tube. These findings dis agree with El-Masri et al., (2009) who reported that the only chest tube related factor was the duration of the chest tube.

The present study found that there was no statistical significance difference between risk factors and size of chest tube. This result in the same line with Rahman et al., (2010) who found that there was no relationship between chest tube size and mortality.

This result disagree with Farhat et al., (2003) who reported that there was no post-operative infections in the small bore group and a 5\% incidence of wound and orifice infection in the regular-sized chest tube group.

This study found that there was highly statistical significance difference between risk factors related to respiratory tract nosocomial infection and chest tube days. Oldfield et al., (2009) in the same line with the current study, where they found that patients whose chest tube days exceeded 20 were almost 6 times more likely to develop HAI than those whose chest tubes days were 20 or less.
Kwiatt et al., (2014) reported that infections at the site of chest tube insertion can travel up the tube to the lungs and cause an internal lung infection. As with any invasion of the body by a piece of tubing, needle or instrument, the risk for infection increases with each passing day that the equipment remains in the body.

\section{Conclusion}

The result of the present study concluded that; the main risk factors for respiratory tract nosocomial infection were contaminated respiratory therapy equipment and patient on mechanical ventilation. Regarding blood stream nosocomial infection, the main risk factors were blood transfusions and improper care of needle insertion site. Failure to use the aseptic technique during dressing was the risk factors for surgical or traumatic wound nosocomial infection. As regards other general risk factors, the main risks were the use of mixed antibiotic, chronic illness, understaffing, overcrowding, smoking, improper hand washing and use of other invasive devices such as (central venous catheter, urinary catheter, and nasogastric tube).

\section{Recommendations}

- Repeat this research on a large sample size and in multi centers for generalization.

- Future studies are recommended that investigate pain management among patient with a chest tube.

- The site of insertion of chest tube must be checked regularly and swabs should be taken to detect any infection early.

- All diagnostic studies and laboratory investigations that needed to detect any infection as CBC should be done for all patients regularly

\section{References}

4. Abd El Hafez G., (2010): The Impact of implementing of standardized nursing care toward the patient with a chest tube to reduce pulmonary complications after Thoracotomy, PP: 58-73.

5. Athanassiadi K, Kakaris S., Theakos N., \& Skottis I., (2007): Muscle-sparing versus posterolateral thoracotomy: a prospective study. Eur J Cardiothorac Surg, Vol. (31), PP: 496-500.

6. Blot S., Koulenti D., \& Dimopoulos G., (2014): EU-VAP Study Investigators. Prevalence, risk factors, and mortality for ventilator-associated pneumonia in middle-aged, old, and very old critically ill patients. Crit Care Med. 2014, Vol. (42), PP: 601-609. 
7. Carlson K., \& Jynn-Mchale D., (2009): AACN procedure Manual for critical Care, 4th ed, Philadelphia: W.B.saunders Co, PP: 123-45.

8. El-Masri M., Oldfield M., \& Fox-Wasylyshyn S., (2009): Examining the association between chest tube-related factors and the risk of developing healthcare-associated infections in the ICU of a community hospital: a retrospective case-control study,Vol. (25),pp:38-44.

9. Eisenberg R., \& Khabbaz K., (2011): Are chest radiographs routinely indicated after chest tube removal following cardiac surgery? American Journal of Roentgenology, 197(1), PP: 122-124.

10. Farhat F., Ginon I., Lefebre M., Andre-Fouet X., \& Mikaeloff P., (2003): Prospective randomized comparison between redon catheters and chest tubes in drainage after cardiac surgery. Journal of Cardiovascular Surgery, 44(2), PP: 179- 186.

11. Gastmeier P., Sohr D., Geffers C., Behnke M., \& Ruden H., (2007): Risk factors for death due to nosocomial infection in intensive care unit patients: findings from the krankenhaus infections surveillance system. Infect Control Hosp Epidemiol. 2007, Vol. (28), PP: 466-472.

12. Gil Z., \& Ryland P., (2014): Tube thoracotomy. Available at http://emedicine.medscape.com. Accessed at March 2016.

13. Humphery-Smith I., (2014): Importance of Bacteriophage in Combating Hospital-Acquired Infection (HAI). Pharmacology \& Pharmacy, Vol. (5), PP: 1192-1201.

14. Kane C., York N., \& Minton L., (2013): Chest tubes in the critically ill patient. Dimensions of Critical Care Nursing, 32(3), PP: 111-117.

15. Kesieme E., Dongo A., Ezemba N., Irekpita E., Jebbin N., \& Kesieme C., (2011): Tube thoracostomy: complications and its management. Pulmonary medicine, 2012, PP: 110.

16. Kwiatt M., Tarbox A., Seamon M., Swaroop M, Cipolla J., Allen C., \& Galwankar S., (2014): Thoracostomy tubes: A comprehensive review of complications and related topics. International journal of critical illness and injury science, 4(2), PP: 143-155.

17. Laith F., Nicole Mallory M., Alfredo Mori M., (2016): Chest tube insertion, American Thoracic Society. Available at:-
Fi/Chest-Tube-Insertion.html accessed at April 2016.

18. Lindgren A., \& Ames N., (2005): caring for a patient on mechanical ventilation: what researches is best practice AJN (2005)105(5):5061.

19. Oldfield M., (2005): Examining the impact of chest tube-related factors on the risk of nosocomial infections in a community-based hospital, Windsor, Ontario, Canada, p: 3.

20. Oldfield M., El-Masri M., \& Fox-Wasylyshyn S., (2009): Examining the association between chest tube-related factors and the risk of developing healthcare-associated infections in the ICU of a community hospital: a retrospective case-control study. Intense \& Crit Care Nurs, Vol. (1), PP: 38-44.

21. Olgac G., Aydogmus U., Mulazimoglu L., \& Kutlu C., (2006): Antibiotics are not needed during tube thoracostomy for spontaneous pneumothorax: an observational case study. J Cardiothorac Surg, 1(1), P: 43.

22. Pekka Ylipalosaari1 \& Tero I., Ala-Kokko., (2006): Intensive care-acquired infection is an independent risk factor for hospital mortality: a prospective cohort study: Critical Care 2006, vol. (10), P: 66.

23. Pollack A., (2010): "Rising Threat of Infections Unfazed by Antibiotics", New York Times, 27, P: 1 .

24. Rahman N., Maskell N., \& Davies C., (2010): The relationship between chest tube size and clinical outcome in pleural infection. Chest 2010, Vol. (137), PP: 536-43.

25. Tukenmez Tigen E., Dogru A., Koltka E., Unlu C., \& Gura M., (2014): Device-associated nosocomial infection rates and distribution of antimicrobial resistance in a medical-surgical intensive care unit in Turkey. Jpn J Infect Dis 2014; 67(1), PP: 5-8. 